\title{
Primum non nocere (first, to do no harm) in prostate biopsy
}

\author{
Peter KF Chiu, MB, ChB, FHKAM (Surgery), CF Ng *, MD FHKAM (Surgery) \\ SH Ho Urology Centre, Department of Surgery, The Chinese University of Hong Kong, Shatin, Hong Kong \\ * Corresponding author: ngcf@surgery.cuhk.edu.hk \\ Hong Kong Med J 2019;25:344-5 \\ https://doi.org/10.12809/hkmj195088
}

Urological symptoms are very common and could present in a wide variety of forms. ${ }^{1,2}$ In this issue of Hong Kong Medical Journal, Cheng et $\mathrm{al}^{3}$ report on emergency attendances and hospitalisations for complications within 30 days after transrectal ultrasound-guided prostate (TRUS) biopsy in two hospitals in Hong Kong. The recorded complications tend to be those that are more severe and require emergency attendances or hospitalisations. Reported rates of sepsis are more accurate as they usually require hospital care. The reported rates of complications such as per rectal bleeding $(0.4 \%)$ and gross haematuria $(2.1 \%)$ were patients that required hospital care, and these are likely much lower than the actual rates. Reported rates of per rectal bleeding and gross haematuria in a systematic review were $11 \%$ to $40 \%$ and $28 \%$ to $64 \%$, respectively. ${ }^{4}$ Although most complications subside within 1 to 2 weeks, there are some potential adverse events that patients should acknowledge. Another point to note is that about $50 \%$ of biopsies in this series had $<10$ biopsy cores taken and this might contribute to a lower complication rate.

Cheng et $\mathrm{al}^{3}$ should be complimented for the low sepsis rate (1.2\% fever, $0.9 \%$ sepsis) after TRUS biopsy, when the rate can be up to $6 \%$ in some series. Transperineal prostate biopsy is an alternative that can achieve near zero sepsis rates, as reported in Caucasian men $^{5}$ and in Chinese men. ${ }^{6}$ There is also no per rectal bleeding in transperineal biopsy. A 'Trexit' initiative to convert all prostate biopsies to transperineal under local anaesthesia has been rolled out in south-east London with the aim to achieve fewer infective complications. ${ }^{7}$ More and more prostate biopsies have been converted to transperineal biopsy in Hong Kong with the aim to eliminate septic complications after prostate biopsy.

In Chinese men with prostate-specific antigen (interquartile range, $5.5-12.6 \mathrm{ng} / \mathrm{mL}$ ), the positive biopsy rate reported by Cheng et al is $19.8 \% .^{3}$ This is much lower than the reported rates of $26 \%$ to $47 \%$ in Caucasian series with prostate-specific antigen $<10$ $\mathrm{ng} / \mathrm{mL} .^{8}$ This is a commonly reported phenomenon among Asian or Chinese men, and indicates that, if most biopsy decisions are based on prostate-specific antigen alone, Chinese or Asian men may undergo more unnecessary biopsies than do Caucasian men. This may raise the question of whether a risk-stratification approach to reduce unnecessary biopsies is more important than improving positive biopsy rates. Using simple and cost-effective tools like prostate cancer risk calculators or blood tests like the prostate health index could reduce unnecessary biopsies and in turn reduce biopsy complications. ${ }^{9,10}$ A multi-parametric magnetic resonance imaging scan of the prostate is also an important tool to improve diagnosis of significant prostate cancer, enable targeted biopsy, and reduce unnecessary biopsies. ${ }^{11}$ However, magnetic resonance imaging is more costly, not easily available in public healthcare setting, and there is a lack of reporting expertise. Furthermore, poorly reported magnetic resonance images with a lot of false positives might also increase unnecessary biopsies. Above all, the principle of primum non nocere (first, to do no harm) should be adhered to; in active surveillance among patients with low-risk prostate cancer, unnecessary biopsies should be avoided. ${ }^{12}$

Finally, using fluoroquinolone as a routine antibiotic prophylaxis for prostate biopsy has been challenged in recent years. Adverse effects of fluoroquinolone antibiotics include tendon ruptures or pain, muscle weakness, numbness or neuropathy, psychiatric adverse events, and life-threatening hypoglycaemia coma in patients with diabetes mellitus. The Food and Drug Administration in the United States issued a drug safety announcement on fluoroquinolones in $2018 .^{13}$ The European Commission issued a legally binding decision in March 2019 on the restriction of use of fluoroquinolone antibiotics, including their use in prevention of recurrent urinary tract infection and in prophylaxis before urological procedures. ${ }^{14}$ This is supported by the European Association of Urology Infections guidelines committee, and fluoroquinolone is expected to be removed from the list of suggested prophylaxis before TRUS prostate biopsy in the next guideline update.

\section{Author contributions}

All authors had full access to the data, contributed to the study, approved the final version for publication, and take responsibility for its accuracy and integrity. 


\section{Conflicts of interest}

The authors have disclosed no conflicts of interest.

\section{Funding/support}

This editorial received no specific grant from any funding agency in the public, commercial, or not-for-profit sectors.

\section{References}

1. Egan KB. The epidemiology of benign prostatic hyperplasia associated with lower urinary tract symptoms: Prevalence and incident rates. Urol Clin North Am 2016;43:289-97.

2. Chan SY, Ng CF, Lee KW, et al. Differences in cancer characteristics of Chinese patients with prostate cancer who present with different symptoms. Hong Kong Med J 2017;23:6-12

3. Cheng KC, Lam WC, Chan HC, et al. Emergency attendances and hospitalisations for complications after transrectal ultrasound-guided prostate biopsies: a 5-year retrospective multicentre study. Hong Kong Med J 2019;25:349-55.

4. Loeb S, Vellekoop A, Ahmed HU, et al. Systematic review of complications of prostate biopsy. Eur Urol 2013;64:876-92.

5. Stefanova V, Buckley R, Flax S, et al. Transperineal prostate biopsies using local anesthesia: Experience with 1,287 patients. Prostate cancer detection rate, complications and patient tolerability. J Urol 2019;201:1121-6.

6. Lo KL, Chui KL, Leung $\mathrm{CH}$, et al. Outcomes of transperineal and transrectal ultrasound-guided prostate biopsy. Hong Kong Med J 2019;25:209-15.

7. The Trexit initiative: transperineal prostate biopsies under local anaesthetic. Available from: nhsaccelerator.com/trexit- initiative-transperineal-prostate-biopsies-local-anaesthetic/. Accessed 24 Jun 2019.

8. Vickers AJ, Cronin AM, Roobol MJ, et al. The relationship between prostate-specific antigen and prostate cancer risk: the Prostate Biopsy Collaborative Group. Clin Cancer Res 2010;16:4374-81.

9. Chiu PK, Alberts AR, Venderbos LD, Bangma CH, Roobol MJ. Additional benefit of using a risk-based selection for prostate biopsy: an analysis of biopsy complications in the Rotterdam section of the European Randomized Study of Screening for Prostate Cancer. BJU Int 2017;120:394-400.

10. Ng CF, Chiu PK, Lam NY, Lam HC, Lee KW, Hou SS. The Prostate Health Index in predicting initial prostate biopsy outcomes in Asian men with prostate-specific antigen levels of 4-10 ng/mL. Int Urol Nephrol 2014;46:711-7.

11. Kasivisvanathan V, Rannikko AS, Borghi M, et al. MRItargeted or standard biopsy for prostate-cancer diagnosis. N Engl J Med 2018;378:1767-77.

12. Tsang CF, Tsu JH, Lai TC, et al. Pathological outcome for Chinese patients with low-risk prostate cancer eligible for active surveillance and undergoing radical prostatectomy: comparison of six different active surveillance protocols. Hong Kong Med J 2017;23:609-15.

13. FDA updates warnings for fluoroquinolone antibiotics on risks of mental health and low blood sugar adverse reactions. Available from: www.fda.gov/news-events/pressannouncements/fda-updates-warnings-fluoroquinoloneantibiotics-risks-mental-health-and-low-blood-sugaradverse. Accessed 24 Jun 2019.

14. European Medicine Agencies. EMA/175398/2019. 11 March 2019. 